\title{
Impact of Cardiac Arrhythmia on Velocity Quantification by ECG gated Phase Contrast MRI
}

\author{
Michael Markl, $\mathrm{PhD}^{1,2}$, Jacob Fluckiger, $\mathrm{PhD}^{1}$, Daniel C. Lee, $\mathbf{M D}^{3}$, Jason $\mathbf{N g}, \mathrm{PhD}^{3}$, and \\ Jeffrey J. Goldberger, MD ${ }^{3,4}$ \\ ${ }^{1}$ Department of Radiology, Feinberg School of Medicine, Northwestern University, Chicago, IL \\ 60611 \\ ${ }^{2}$ Department of Biomedical Engineering, McCormick School of Engineering, Northwestern \\ University, 2145 Sheridan Road, Evanston, IL 60208 \\ ${ }^{3}$ Division of Cardiology, Northwestern University, Chicago, IL 60611 \\ ${ }^{4}$ Section of Cardiac Electrophysiology, Northwestern University, Chicago, IL 60611
}

\begin{abstract}
Objective-To systematically investigate the impact of beat-to-beat variations on ECG gated multi-beat flow imaging with phase contrast (PC) MRI based on real time in-vivo TEE data in patients with known arrhythmia.
\end{abstract}

Methods-Real-time 2D Doppler transesophageal echocardiography (TEE) was performed in five patients with atrial fibrillation ( 4 male, age $=64 \pm 8.7$ years). TEE data provided real-time left atrial (LA) and left ventricular (LV) flow velocities in consecutive cardiac cycles with different RR-interval durations. PC-MRI acquisitions were simulated from the TEE velocity measures by constructing time-resolved $\mathrm{k}$-space data for segmented sampling schemes typically used for ECG gated 2D PC MRI. Each simulation was repeated 100 times to minimize effects from data that may be weighted to a particular beat in the center of k-space. The resulting LA and LV velocities were compared to the average TEE velocities and data from individual cardiac cycles.

Results-Despite beat-to-beat variations velocities in TEE data, ECG gated flow imaging with MRI could reproduce persistent average LA and LV mean velocities within 7.0-7.4\% compared to TEE.

Conclusions-PC-MRI velocity measurements in patients with varying R-R interval durations are not significantly different from time-averaged real-time velocity data for a typical segmented $\mathrm{k}$-space data acquisition schemes. Though beat-to-beat variations in atrial velocities that were observed with TEE cannot be detected with ECG gated multi-beat PC MRI, it can reliably assess average flow patterns across multiple beats

\section{Keywords}

Phase contrast MRI; blood flow; arrhythmia; atrial fibrillation

Corresponding author: Michael Markl, PhD, Departments of Radiology and Biomedical Engineering, Northwestern University Feinberg School of Medicine, 737 N. Michigan Avenue Suite 1600, Chicago, Illinois 60611, USA, Phone: +1 312-695-1799, mmarkl@northwestren.edu. 


\section{Introduction}

Phase contrast (PC) MRI is widely used for the measurement of cardiovascular blood flow ${ }^{1-4}$ and is typically prospectively or retrospectively gated to the ECG signal ${ }^{5}$. Data are usually acquired over several heart beats using segmented $\mathrm{k}$-space sampling. The resulting images represent a composite view of blood flow over the entire acquisition period while beat-to-beat variations are assumed to be small. In patients with arrhythmic heart rates, however, variations in the length of the cardiac cycle during the course of the MR acquisition can be substantial. In fact, cardiac arrhythmia is often seen in patients with atrial fibrillation (AF) which is a common cardiac abnormality with high prevalence ${ }^{6}$. AF is characterized by irregular activation of the atria which can lead to a number of complications including high risk of stroke due left atrial thromboembolism (it is estimated that $15-20 \%$ of all strokes occur in patients with AF) ${ }^{7}$. Previous studies with Doppler transesophageal echocardiography (TEE) have shown that patients with AF demonstrate reduced blood flow velocity in the left atrium (LA), which may be a contributing factor to thrombus formation ${ }^{8-10}$. However, due to the need for esophageal intubation and patient sedation to enable TEE acquisition, a less invasive alternative for measuring LA blood flow in AF patients is desirable.

PC MRI may be a non-invasive alternative to quantify left atrial velocities and thus assess stroke risk ${ }^{11}$. However, the total acquisition time typically spans multiple cardiac cycles and thus may include data from cardiac cycles with large deviation in cycle length and beatto-beat variations in blood flow velocities. Several real-time MR acquisition strategies have recently been developed for cardiovascular imaging ${ }^{12-14}$, though these strategies may not be immediately available for clinical use. It is thus desirable to understand the impact of cardiac arrhythmia on existing, ECG-gated PC-MRI acquisitions.

The aim of this study was to use real-time in vivo Doppler TEE data acquired in patients in AF to simulate the complex k-space-weighted averaging in a 2D PC-MRI acquisition. We performed simulations investigating multiple k-space acquisition schemes typically used in 2D PC-MRI. The goal was to test the hypothesis that 2D PC-MRI in patients with cardiac arrhythmia can reliably assess patterns of blood flow velocities that are consistently present over multiple heart beats.

\section{Methods}

\section{TEE Data Acquisition}

Doppler TEE was performed in 5 patients ( 4 male, mean age $=64 \pm 8.7$ years) using an $\mathrm{X} 7-2 \mathrm{t}$ transducer (iE33, Philips Medical Systems). All patients were in atrial fibrillation at the time of imaging. Each patient was fasted prior to imaging, and was sedated with intravenous midazolam and fentanyl. Two-dimensional color flow Doppler images of the mitral valve were obtained in the standard mid-esophageal four chamber view with isotropic spatial resolution of $0.15 \mathrm{~mm}$ and a mean frame rate of 14 frames per second. The color flow images represented single-direction velocity encoding along the axis of the TEE probe. Two to four consecutive heartbeats of varying length were acquired for each patient. This retrospective 
study was approved by our institution's review board. Details on the acquired TEE data (number of cardiac cycles available for analysis, duration of each RR-interval) are shown in Table 1.

\section{TEE based 2D PC-MRI Simulations}

The strategy for the TEE-based simulation of ECG gated PC-MRI data is outlined in figure 1. For each patient, real time Color Doppler TEE data of the LA and left ventricle (LV) over several heart beats were available. Figure 1A shows an example of a TEE time frame for 3 consecutive RR-intervals. For each RR interval and time frame, the TEE image was first converted to gray scale to generate the magnitude image for the MR simulations. In addition, velocity values for each voxel in the Doppler TEE data were extracted. For each patient, the maximum absolute velocity value was identified as $\mathrm{v}_{\max }$ and the velocity values were normalized by $\pi / \mathrm{v}_{\max }$ to represent the velocity-encoded phase image (figure 1B). Based on the magnitude and phase images, 2D dimensional Fourier transform was employed to simulate the complex MR k-space data for this particular RR-interval and time frame. Conversion of all TEE data (multiple RR-intervals of different lengths) resulted in a timeresolved series of TEE derived real-time k-space data that spanned several heart beats (figure 1C).

The TEE derived k-space data was then used to simulate k-space segmented 2D PC-MRI data acquired over multiple cardiac cycles (figure 1D). The simulated resolution, field of view, and temporal resolution were identical to that in the original TEE data. Typical TEE parameters included a spatial resolution of $0.2 \mathrm{~mm}$ in-plane resolution, a $200 \times 160 \mathrm{~mm}$ field of view, and a temporal resolution of $71 \mathrm{~ms}$ per time frame. For 2D PC-MRI simulations kspace segmentation factors of $\mathrm{N}_{\mathrm{Seg}}=4$ and $\mathrm{N}_{\mathrm{Seg}}=1$ was assumed, i.e. lines were collected for each RR-interval and data acquisition was continued over multiple heartbeats until all phase encodings were acquired. As illustrated in figure 2, three separate k-space filling schemes were simulated: (MRI 1) k-space was divided into 4 regions and for each simulated heartbeat, one line of $\mathrm{k}$-space was put into each of the segments until all segments were filled; (MRI 2) for each simulated heartbeat four consecutive lines of $k$-space were filled until all k-space was collected; (MRI 3) only one line of k-space was acquired per heartbeat. Sampling strategies MRI 1 and MRI 2 represent k-space filling patterns typically used for standard 2D PC-MRI protocols.

To simulate data acquisition with beat to beat variations, 2D PC-MRI k-space data was constructed by selecting $\mathrm{k}$-space data from different RR-intervals of the TEE derived $\mathrm{k}$ space data. For each simulated PC-MRI ECG cycle, a TEE RR-interval was randomly selected and the $\mathrm{N}_{\text {Seg }} \mathrm{k}$-space lines were copied to the MRI k-space until the 2D PC-MRI kspace was filled. The composite 2D PC-MRI k-space data were then transformed with an inverse Fourier transform to obtain simulated MR velocity and magnitude images (figure $1 \mathrm{E})$.

Due to the random nature of the simulations, each was repeated 100 times to minimize effects from data that may be weighted to a particular beat in the center of k-space. The average simulated MR velocity and magnitude were calculated for each $\mathrm{k}$-space acquisition 
methods MRI 1, MRI 2, and MRI 3. For comparison, the mean velocity from the TEE data over all acquired RR-intervals was calculated.

\section{Data Analysis}

Results from the 2D PC-MRI simulations were compared with the individual (each RRinterval) and average TEE velocities. The average velocity measurements in the PC-MRI simulations were quantitatively compared to the TEE data using a region of interest (ROI) approach. Two ROIs were manually drawn on each TEE dataset by an experienced observer. One ROI was placed within the left atrium, and the other was placed over the mitral valve and extending into the left ventricle (see figure 5). These ROIs were then copied onto the simulated PC-MRI data for each time frame. The ROIs were used to calculate histograms showing the velocity distribution in the ROIs for the MR simulation, for the average TEE data, and for each individual TEE-measured heartbeat. In addition, the mean velocity and the incidence, i.e. normalized number of voxels, with atrial velocities greater than a threshold of $20 \mathrm{~cm} / \mathrm{s}$ were calculated. The selection of the velocity threshold was based on previous TEE results which found that systolic atrial velocities less than $20 \mathrm{~cm} / \mathrm{s}$ constitute a risk factor thrombus formation and for stroke ${ }^{15,16}$.

\section{Results TEE Data}

The average $\mathrm{R}-\mathrm{R}$ interval duration for all five patients was $643 \pm 161 \mathrm{~ms}$, with a range of $364-1000 \mathrm{~ms}$ and a total number of 2-4 RR-intervals were acquitted for each patient (table 1). A representative frame of TEE data for one patient at systole and diastole over three consecutive heartbeats is shown in figure 3. The durations of the R-R intervals for these three beats were $750 \mathrm{~ms}, 660 \mathrm{~ms}$, and $580 \mathrm{~ms}$, respectively. At peak systole (top row), a large mitral regurgitation jet can be seen over all three heartbeats. The bottom row shows a single frame from the late diastolic portion of the heartbeat. Appreciable variations in beat-to-beat velocity are evident. Similar results were obtained in all five patients and showed consistent spatial distribution of blood velocity during peak systole over all heartbeats.

\section{D PC-MRI Simulations}

Representative results of the simulation of 2D PC-MRI velocity measurements compared to TEE data at both peak systole (top row), and late diastole (bottom row) from one patient are depicted in figure 4 . The TEE velocity data was averaged over all acquired heartbeats and is compared to the mean composite MRI velocity from all 100 simulations for the three different k-space sampling schemes (MRI 1, 2, and 3). The spatial distribution of the positive and negative velocity at both systole and diastole was consistent between the TEE and MR data.

Table 2 summarizes the cumulative results of the 2D PC MRI simulations for mean velocities (averaged of the LA and LV ROIs) and incidence compared to findings from cycle averaged Doppler TEE data. Despite beat-to-beat variations, 2D PC-MRI simulations resulted in similar mean velocities with only small differences compared to TEE of 7.0 $\pm 6.6 \%$ for MRI $1,7.4 \pm 8.4 \%$ for MRI 2, 7.3 $\pm 6.6 \%$ for MRI 3 for all $\mathrm{k}$-space filling 
patterns. For incidence, differences were more elevated (12.0 $\pm 10.2 \%$ for MRI 1, $17.6 \pm 13.5 \%$ for MRI $2,11.0 \pm 10.8 \%$ for MRI 3). The variation in mean velocity between the three MR simulation methods was less than $10 \%$ for all patients. Average TEE and simulated MR velocity measurements within the ROIs were not significantly different.

\section{Discussion}

PC-MRI allows for non-invasive, time-resolved measurement of blood velocity. Although previous work with PC-MRI in patients with AF suggests that atrial fibrillation leads to decreased blood velocity and increased flow incoherence in the left atrium ${ }^{11}$, to our knowledge, the impact of beat-to-beat variations on the resulting 2D PC-MRI data has not been systematically investigated to date.

In this work we simulated a 2D PC-MRI acquisition with single-direction in-plane velocity encoding in patients with AF using real time TEE data. The results presented here suggest that filling $\mathrm{k}$-space with data acquired from heartbeats of various durations returns velocity values similar to what would be obtained by averaging the velocities over the total length of the acquisition. The the three acquisition schemes tested here (MRI 1-3) resulted in similar MRI velocity data and returned mean left atrial and ventricular velocities that were within $7.0-7.4 \%$ of the mean velocity measured with TEE. Though beat-to-beat variations in atrial and ventricular velocities that were observed with TTE cannot be detected with ECG gated 2D PC-MRI, it was possible to reliably measure persistent average velocity patterns (i.e. velocities that are common to most cardiac cycles).

In patients with $\mathrm{AF}$, one of the greatest risks is thrombus formation in the left atrium due to low-velocity flow or regions of stasis. We speculate that in patients with AF or other cardiac arrhythmia the persistent average flow patterns likely have more physiological significance ${ }^{16}$ since thrombus formation is thought to occur in regions with consistently low blood flow velocities. This suggests that PC-MRI has the potential to be a useful tool in the assessment of persistent or average flow characteristics and thus stroke risk in patients with atrial fibrillation and other cardiac arrhythmias. Though several different risk scores have been used to evaluate stroke risk in patients with atrial fibrillation, recent studies have shown that the discerning power of these scores is limited at best ${ }^{17}$. These risk scores are generally based on global epidemiologic factors such as age, hypertension, diabetes mellitus, and history of stroke but do not assess underlying mechanisms of thrombus formation such as left atrial flow. PC-MRI, in contrast, is able to investigate average flow patterns over the length of the acquisition, which may influence thrombus formation.

The simulations performed here were limited to a single slice of data with a single, in-plane, velocity encoding. It should be noted that typical PC-MR imaging focuses more on throughplane velocity encoding to measure volume flow. In addition, much recent work has been done using time resolved, three-dimensional PC-MRI with three-directional velocity encoding, known as 4D flow MRI ${ }^{5}{ }^{18-20}$. Though extending these simulations to a threedimensional volume would likely not change the results, future work should focus on examining the averaging effects of a 4D flow MRI acquisition with three-directional velocity encoding. We note that the conversion of the TEE data to simulated MRI data is not 
identical to the images obtained in a true PC-MRI acquisition. The magnitude images are obscured in regions by the overlying velocity information on the TEE images. However, this will not impact the results of the simulations as the magnitude images are used only for anatomical reference. Similarly, true PC-MRI acquires reference and velocity-encoded images separately and then calculates the phase difference between the two while this simulation uses only a single, velocity-encoded phase image.

One additional limitation of this study is the limited number of RR-intervals available for generating the MR simulations. In this work, we used multiple realizations of the simulation with random selection of $\mathrm{k}$-space lines used to generate the composite PC-MRI images. This was done in an attempt to mimic the irregularity of the heartbeat in patients in AF. In practice, a time-resolved, three-dimensional PC-MRI scan may acquire data over several minutes, covering several hundred heartbeats. In addition, the number of patients used in this study is small, which limits the statistical power of the results presented here.

\section{Conclusion}

PC-MRI has the potential to evaluate persistent average, or typical flow patterns in the heart and major vessels even in patients with cardiac arrhythmia. Future work with PC-MRI in patients with arrhythmia should focus on validating 4D flow velocity measurements in vivo in a three-dimensional volume with three-direction velocity encoding and with a larger cohort of patients.

\section{Acknowledgments}

Grant support: AHA grant-in-aid 12GRNT12080032, NIH NHLBI grant R21 HL113895.

\section{References}

1. Mohiaddin RH, Pennell DJ. Mr Blood Flow Measurement. Clinical Application in the Heart and Circulation. Cardiol Clin. 1998; 16:161-187. [PubMed: 9627755]

2. Chai P, Mohiaddin R. How We Perform Cardiovascular Magnetic Resonance Flow Assessment Using Phase-Contrast Velocity Mapping. J Cardiovasc Magn Reson. 2005; 7:705-716. [PubMed: 16136862]

3. Didier D. Assessment of Valve Disease: Qualitative and Quantitative. Magn Reson Imaging Clin N Am. 2003; 11:115-134. vii. [PubMed: 12797514]

4. Gatehouse PD, Keegan J, Crowe LA, Masood S, Mohiaddin RH, Kreitner KF, Firmin DN. Applications of Phase-Contrast Flow and Velocity Imaging in Cardiovascular Mri. Eur Radiol. 2005; 15:2172-2184. [PubMed: 16003509]

5. Markl M, Frydrychowicz A, Kozerke S, Hope MD, Wieben O. 4d Flow Mri. J Magn Reson Imaging. 2012 in press.

6. Feinberg WM, Blackshear JL, Laupacis A, Kronmal R, Hart RG. Prevalence, Age Distribution, and Gender of Patients with Atrial Fibrillation. Analysis and Implications. Arch Intern Med. 1995; 155:469-473. [PubMed: 7864703]

7. Singer DE, Albers GW, Dalen JE, Fang MC, Go AS, Halperin JL, Lip GY, Manning WJ. Antithrombotic Therapy in Atrial Fibrillation: American College of Chest Physicians EvidenceBased Clinical Practice Guidelines (8th Edition). Chest. 2008; 133:546S-592S. [PubMed: 18574273] 
8. The Stroke Prevention in Atrial Fibrillation Investigators Committee on Echocardiography. Transesophageal Echocardiographic Correlates of Thromboembolism in High-Risk Patients with Nonvalvular Atrial Fibrillation. Ann Intern Med. 1998; 128:639-647. [PubMed: 9537937]

9. Goldman ME, Pearce LA, Hart RG, Zabalgoitia M, Asinger RW, Safford R, Halperin JL. Pathophysiologic Correlates of Thromboembolism in Nonvalvular Atrial Fibrillation: I. Reduced Flow Velocity in the Left Atrial Appendage (the Stroke Prevention in Atrial Fibrillation [Spaf-Iii] Study). J Am Soc Echocardiogr. 1999; 12:1080-1087. [PubMed: 10588784]

10. Fatkin D, Kelly RP, Feneley MP. Relations between Left Atrial Appendage Blood Flow Velocity, Spontaneous Echocardiographic Contrast and Thromboembolic Risk in Vivo. J Am Coll Cardiol. 1994; 23:961-969. [PubMed: 8106703]

11. Fluckiger JU, Goldberger JJ, Lee DC, Ng J, Lee R, Goyal A, Markl M. Left Atrial Flow Velocity Distribution and Flow Coherence Using Four-Dimensional Flow Mri: A Pilot Study Investigating the Impact of Age and Pre- and Postintervention Atrial Fibrillation on Atrial Hemodynamics. J Magn Reson Imaging. 2013; 38:580-587. [PubMed: 23292793]

12. Zhang S, Uecker M, Voit D, Merboldt KD, Frahm J. Real-Time Cardiovascular Magnetic Resonance at High Temporal Resolution: Radial Flash with Nonlinear Inverse Reconstruction. J Cardiovasc Magn Reson. 2010; 12:39. [PubMed: 20615228]

13. Nayak KS, Hu BS. The Future of Real-Time Cardiac Magnetic Resonance Imaging. Curr Cardiol Rep. 2005; 7:45-51. [PubMed: 15610648]

14. Kowalik GT, Steeden JA, Pandya B, Odille F, Atkinson D, Taylor A, Muthurangu V. Real-Time Flow with Fast Gpu Reconstruction for Continuous Assessment of Cardiac Output. J Magn Reson Imaging. 2012; 36:1477-1482. [PubMed: 22745017]

15. Bernhardt P, Schmidt H, Hammerstingl C, Luderitz B, Omran H. Patients with Atrial Fibrillation and Dense Spontaneous Echo Contrast at High Risk a Prospective and Serial Follow-up over 12 Months with Transesophageal Echocardiography and Cerebral Magnetic Resonance Imaging. J Am Coll Cardiol. 2005; 45:1807-1812. [PubMed: 15936610]

16. Baumgartner H, Hung J, Bermejo J, Chambers JB, Evangelista A, Griffin BP, Iung B, Otto CM, Pellikka PA, Quinones M. Echocardiographic Assessment of Valve Stenosis: Eae/Ase Recommendations for Clinical Practice. J Am Soc Echocardiogr. 2009; 22:1-23. quiz 101-102. [PubMed: 19130998]

17. Lip GY, Nieuwlaat R, Pisters R, Lane DA, Crijns HJ. Refining Clinical Risk Stratification for Predicting Stroke and Thromboembolism in Atrial Fibrillation Using a Novel Risk Factor-Based Approach: The Euro Heart Survey on Atrial Fibrillation. Chest. 2010; 137:263-272. [PubMed: 19762550]

18. Frydrychowicz A, Harloff A, Jung B, Zaitsev M, Weigang E, Bley TA, Langer M, Hennig J, Markl M. Time-Resolved, 3-Dimensional Magnetic Resonance Flow Analysis at 3 T: Visualization of Normal and Pathological Aortic Vascular Hemodynamics. J Comput Assist Tomogr. 2007; 31:915. [PubMed: 17259827]

19. Mark1 M, Benk C, Klausmann D, Stalder AF, Frydrychowicz A, Hennig J, Beyersdorf F. ThreeDimensional Magnetic Resonance Flow Analysis in a Ventricular Assist Device. J Thorac Cardiovasc Surg. 2007; 134:1471-1476. [PubMed: 18023667]

20. Markl M, Geiger J, Arnold R, Stroh A, Damjanovic D, Foll D, Beyersdorf F. Comprehensive 4Dimensional Magnetic Resonance Flow Analysis after Successful Heart Transplantation Resolves Controversial Intraoperative Findings and Reveals Complex Hemodynamic Alterations. Circulation. 2011; 123:e381-383. [PubMed: 21422394] 


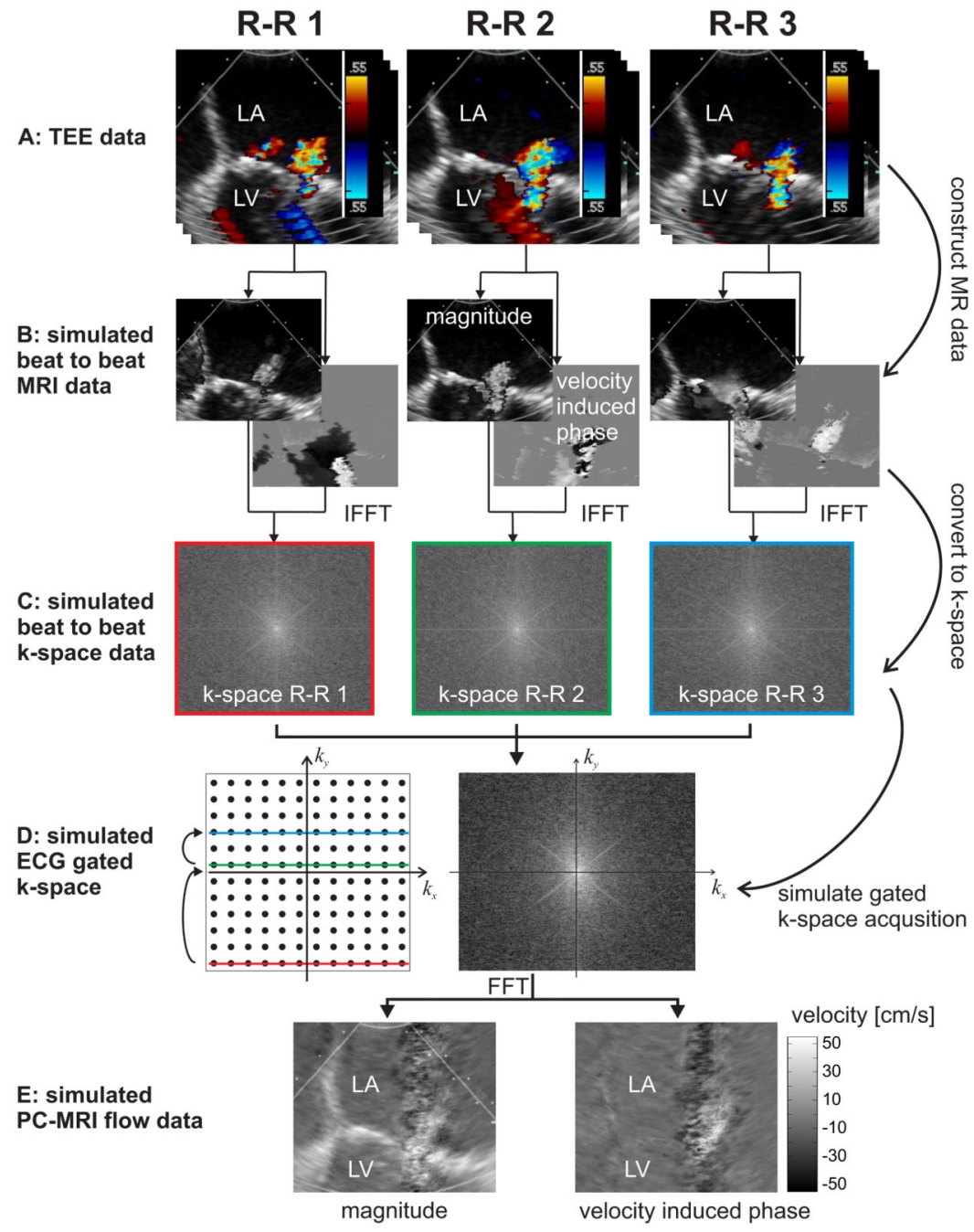

Figure 1.

Flowchart of the MR simulation methodology. Original TEE data (A) are separated into RRintervals and converted to grayscale (B, left), and velocity measures are extracted from the color Doppler images (B, right). Each complex image (magnitude: grayscale, phase: velocity) undergoes a Fourier transform to simulate MRI k-space (C). K-space lines are randomly selected using one of three acquisition schemes (sample selection from three heartbeats shown in (C) in (D, left)) to create 2D PC MRI k-space data (D., right), which undergoes an inverse Fourier transform to calculate a simulated MR reconstruction of 2D PC MRI magnitude and velocity images (E). LA: left atrium, LV: left ventricle. 

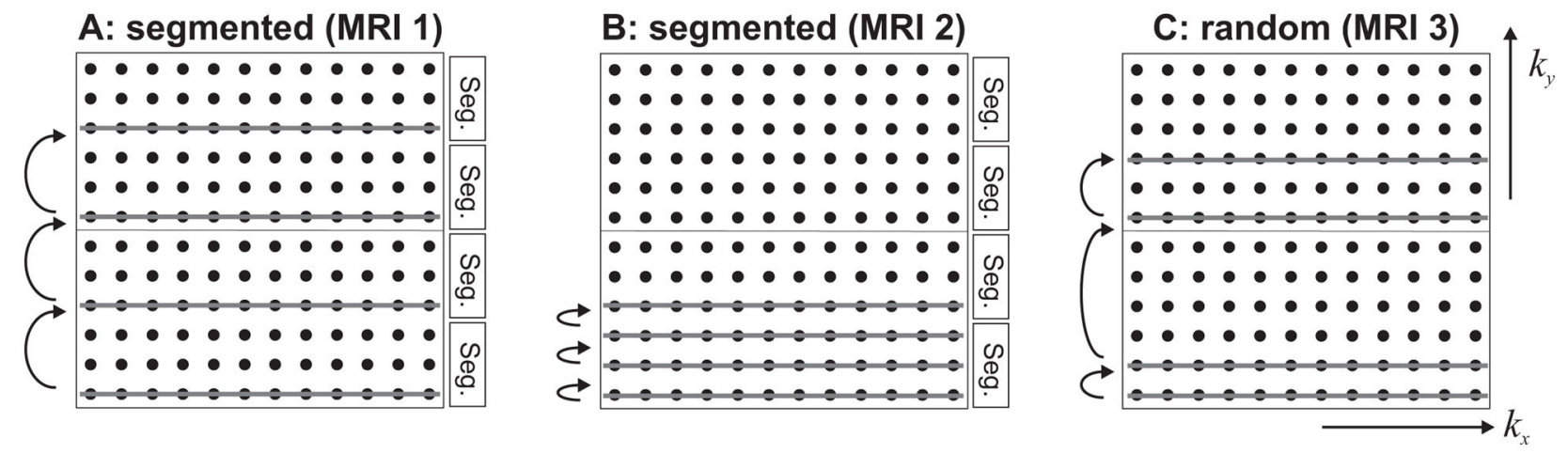

Figure 2.

K-space acquisition sampling patterns used in 2D PC-MRI simulations. In MRI 1, k-space was divided into 4 segments, with one line filled in each segment per heartbeat. In MRI 2 , four consecutive lines of k-space are filled for each heartbeat. In MRI 3, four lines of kspace are placed randomly into unfilled locations per heartbeat. 


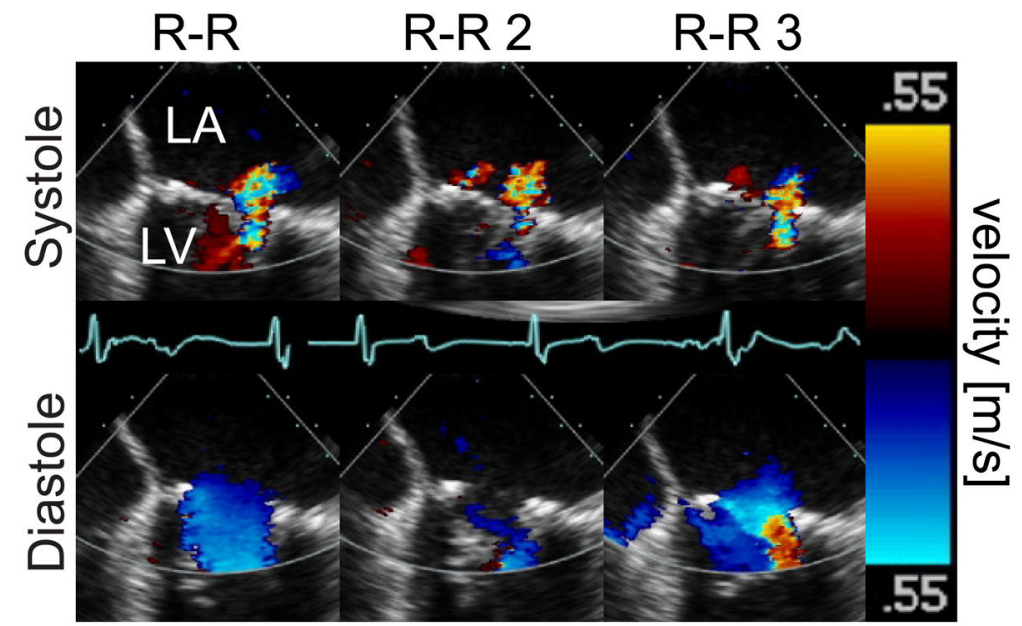

Figure 3.

Representative TEE data for a single patient with atrial fibrillation at systole (top row), and diastole (bottom row) for three consecutive heartbeats (R-R intervals of $750 \mathrm{~ms}, 660 \mathrm{~ms}$, and 580ms). LA: left atrium, LV: left ventricle 


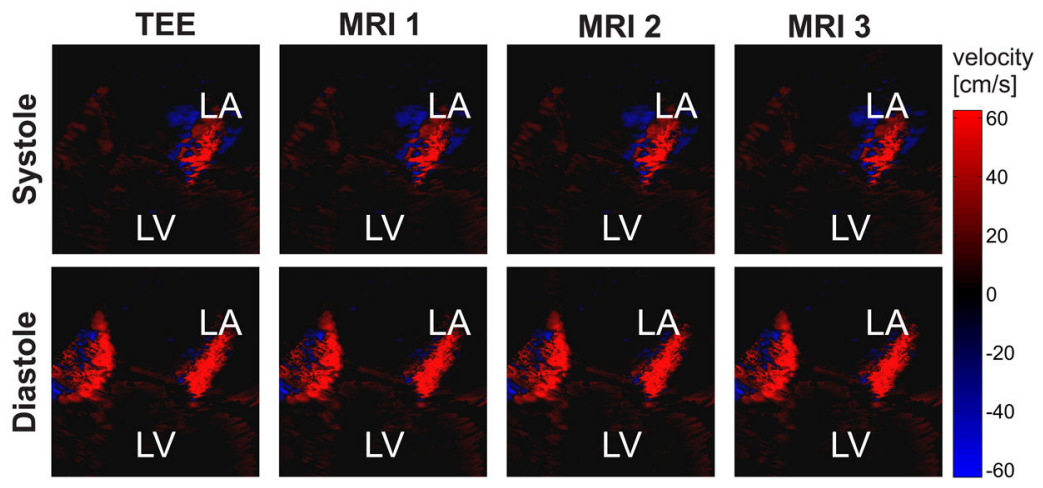

Figure 4.

Average TEE data over all acquired heartbeats for one representative atrial fibrillation patients at systole (top row) and diastole (bottom row). The reconstructed velocity images from the MR simulations are also shown. MRI 1-3 denote the 3 different k-space sampling schemes used for the simulation of prospectively gated 2D PC-MRI data. LA: left atrium, LV: left ventricle. 


\section{Table 1}

Number of R-R intervals and R-R interval durations for each patient

\begin{tabular}{ccc}
\hline Patient & Number of RR Intervals & RR Interval Durations [ms] \\
\hline 1 & 4 & $546,364,455,636$ \\
2 & 3 & $750,750,583$ \\
3 & 2 & 1000,714 \\
4 & 3 & $737,474,632$ \\
5 & 3 & $750,750,500$ \\
\hline
\end{tabular}

竞

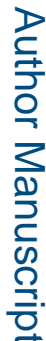

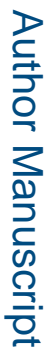

J Comput Assist Tomogr. Author manuscript; available in PMC 2016 May 01. 


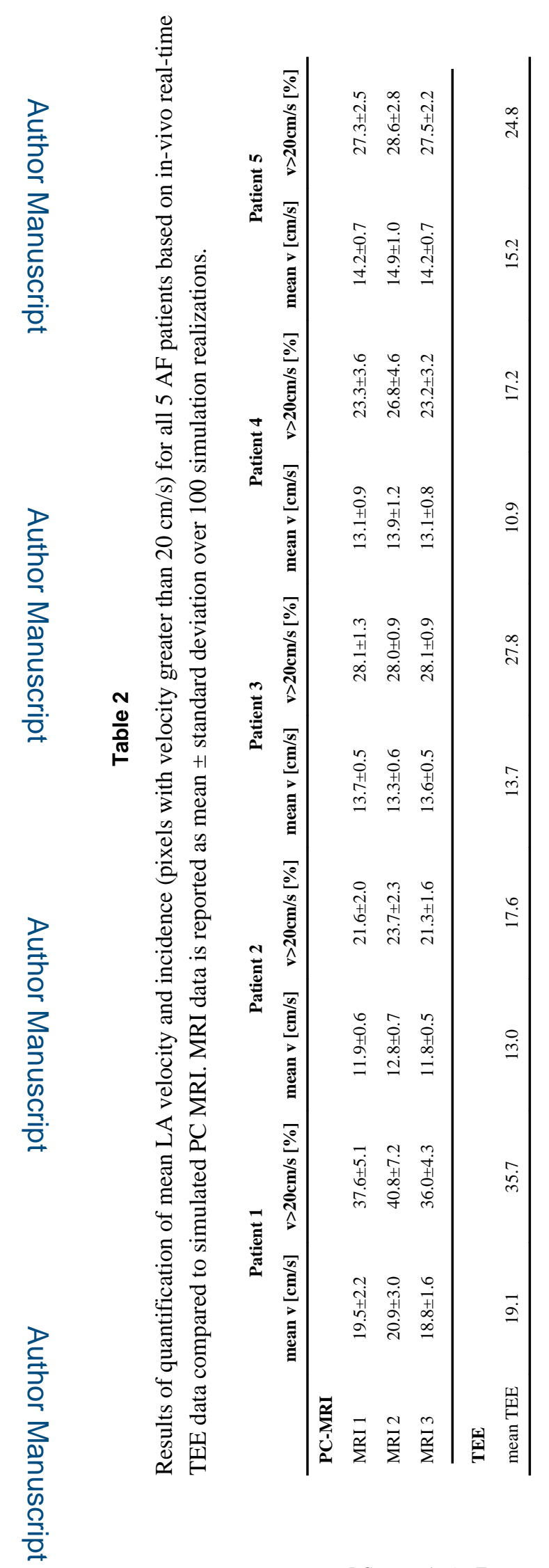

J Comput Assist Tomogr. Author manuscript; available in PMC 2016 May 01. 